\title{
Anatomically-Induced Fibrillation in a 3D Model of the Human Atria
}

\author{
Mark Potse ${ }^{1,2,3}$, Ali Gharaviri ${ }^{4}$, Simone Pezzuto $^{4}$, Angelo Auricchio $^{4}$, Rolf Krause ${ }^{4}$, \\ Sander Verheule ${ }^{5}$, Ulrich Schotten ${ }^{5,4}$ \\ ${ }^{1}$ CARMEN Research Team, Inria Bordeaux Sud-Ouest, Talence, France \\ ${ }^{2}$ IHU Liryc, fondation Bordeaux Université, Pessac, France \\ ${ }^{3}$ Université de Bordeaux, IMB, UMR 5251, Talence, France \\ ${ }^{4}$ Center for Computational Medicine in Cardiology, Lugano, Switzerland \\ ${ }^{5}$ Department of Physiology, Maastricht University, Maastricht, The Netherlands
}

\begin{abstract}
Atrial fibrillation $(A F)$ requires both a trigger and a substrate that can maintain a complex reentrant activity. In patients and in experimental models this substrate is provided by both electrical and structural remodeling. Since these processes overlap in time it is impossible to assess their individual contributions to AF maintenance experimentally. Therefore we studied the effect of electrical remodeling alone on AF initiation in a realistic numerical model of the human atria. We attempted to initiate AF by rapid pacing in 10 different locations, both with and without electrical remodeling. The protocols were repeated twice, with small variations in calcium conductivity, so that in total 30 simulations with and 30 simulations without remodeling were performed. In models with electrical remodeling, functional conduction block at structural inhomogeneities induced AF in $27 \%$ of the simulations. In models without electrical remodeling, AF could not be induced. We conclude that in the complex anatomy of the atria electrical remodeling alone increases the probability of AF substantially. This finding supports a mechanism whereby electrical remodeling, which occurs relatively fast, accelerates the slower but irreversible structural remodeling process.
\end{abstract}

\section{Introduction}

Atrial fibrillation (AF) requires a substrate and a trigger. The substrate is a stretch of myocardium that can accomodate a reentrant wave. Slow propagation, a tortuous route, and a short refractory period can contribute to such a substrate. The trigger may be an ectopic impulse that arrives during a vulnerable window, or a situation of unidirectional conduction block. Ectopic impulses often arise from the pulmonary veins [1], and may themselves be caused by reentrant activity, on a microscopic scale [2].
Rapid ectopic or paced activity in the atria promotes an arrhythmic substrate by inducing both electrical and structural remodeling [3]. The electrical remodeling process sets in first and involves changes in calcium and potassium currents that shorten the action potential and thus the wavelength. Structural remodeling, consisting mainly of interstitial fibrosis, develops more slowly, and contributes to AF vulnerability by slowing conduction and creating obstacles. Thus, electrical and structural remodeling have a common cause, but it is not as firmly established that electrical remodeling alone can cause AF. For example, apart from initial structural remodeling, repolarization heterogeneity may also play a role $[4,5]$. The purpose of this study is therefore to test the effect of homogeneous electrical remodeling alone, in a realistic numerical model of the human atria.

\section{Methods}

We used a realistic atrial anatomy prepared by a combination of imaging and literature data, as described previously [6], but with a more realistic multiple-layer fiber structure in the left atrium, posterior inter-atrial bundles, and a few fiber tracts connecting the left atrium with the coronary sinus musculature (figure 2). These features were manually edited based on literature [7-9]. From these elements, illustrated in figure $2 \mathrm{~A}$, a computational mesh was created algorithmically. This mesh consisted of approximately 5 million nodes with $200 \mu \mathrm{m}$ spacing. Outside the thicker bundles, the atrial wall was between 2 and $3 \mathrm{~mm}$ thick, i.e. about 10 to 15 model elements.

Electrical activity was simulated with a monodomain reaction-diffusion equation using the human atrial myocyte model developed by Courtemanche et al. [10]. While our model uses several different tissue conductivity values (Table 1), the parameters of the ionic model were uniform. Simulations were performed with the Propag-5 code [11] 


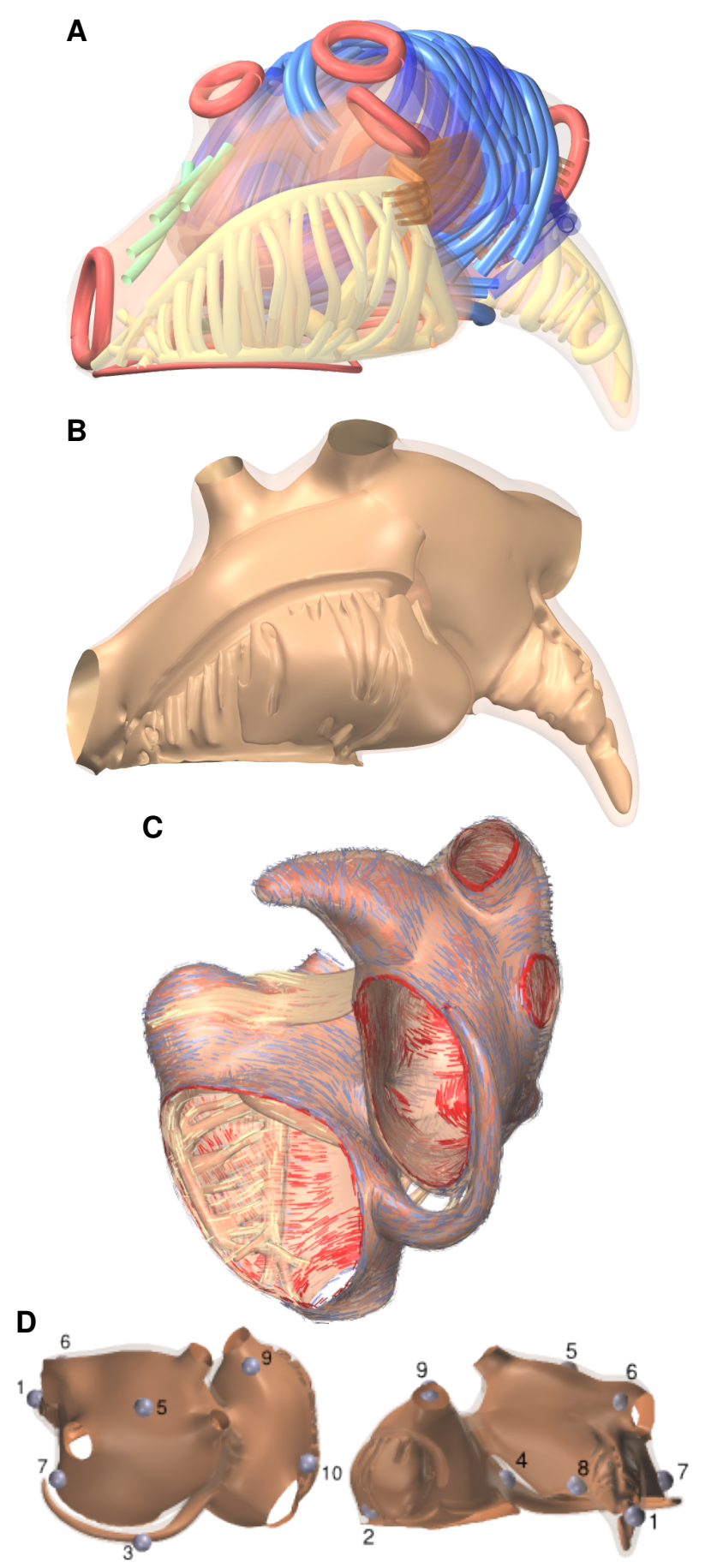

Figure 1. A: Imaging-derived outline and manually-edited curves to define bundles and fiber orientations in the atria. The yellow tubes define the crista terminalis, pectinate muscles, and other bundles. Green tubes define posterior interatrial bundles. Red and blue tubes define fiber orientations; dark blue for the inner layer and light blue for the outer layer of the left atrium. B: The resulting thickness variations in the atrial wall. C: The resulting fiber orientations (red, endocardial; blue, epicardial; yellow, bundle). D: The 10 randomly chosen stimulus locations.

\begin{tabular}{lcccccc} 
material & $\sigma_{\mathrm{iL}}$ & $\sigma_{\mathrm{iT}}$ & $\sigma_{\mathrm{iC}}$ & $\sigma_{\mathrm{eL}}$ & $\sigma_{\mathrm{eT}}$ & $\sigma_{\mathrm{eC}}$ \\
\hline anisotropic & 3.0 & 0.3 & 0.3 & 3.0 & 1.2 & 1.2 \\
isotropic & 1.5 & 1.5 & 1.5 & 1.5 & 1.5 & 1.5 \\
$\begin{array}{l}\text { Bachmann's } \\
\text { bundle }\end{array}$ & 9.0 & 0.3 & 0.3 & 9.0 & 1.2 & 1.2
\end{tabular}

Table 1. Tissue conductivities $(\sigma)$ in $\mathrm{mS} / \mathrm{cm}$. The subscript 'i' stands for intracellular, 'e' for extracellular, ' $\mathrm{L}$ ' for longitudinal, ' $\mathrm{T}$ ' for transverse (within a tissue sheet) and ' $\mathrm{C}$ ' for cross-sheet. Most of the model elements had the anisotropic type. The isotropic tissue type was used in a small area between the caval veins.

\begin{tabular}{lll} 
& normal & remodeled \\
\hline$g_{\mathrm{CaL}}$ & 0.1238 & 0.037 \\
$g_{\mathrm{TO}}$ & 0.1652 & 0.026 \\
$g_{\mathrm{K} 1}$ & 0.09 & 0.180 \\
$g_{\mathrm{Ks}}$ & 0.129 & 0.1 \\
$g_{\mathrm{Na}}$ & 7.8 & 8.0
\end{tabular}

Table 2. Maximum conductivities of ionic currents $(\mathrm{nS} / \mathrm{pF})$ for simulations of normal and remodeled atria.

and ran on either 2048 cores of a Bullx cluster machine or on 4096 cores of an IBM BlueGene/Q.

To simulate normal atria, we used the original parameter values of the ionic model [10]. To simulate remodeled atria we modified the maximum conductivities of the calcium current, $g_{\mathrm{CaL}}$, and three potassium currents as detailed in Table 2 [12]. To test the sensitivity of our results to small changes in model parameters we repeated the simulations with $101 \%$ and $102 \%$ of the $g_{\mathrm{CaL}}$ values given in the table, for both normal and remodeled atria.

In each simulation, 14 stimuli were delivered at the same location with an interval decreasing from 280 to $124 \mathrm{~ms}$. For each parameter set 10 simulations were performed, each with a different stimulus location (figure 2D). The 10 stimulus locations were chosen randomly, but guaranteed to be at least $30 \mathrm{~mm}$ apart.

\section{Results}

Figure 2 shows an example of a succesful AF initiation. The atria were stimulated from the left atrial appendage (site 1) and $g_{\mathrm{CaL}}$ was $101 \%$ of the "remodeled" value (Table 2). A reentry developed due to functional block at the narrow connection between the left atrium and the coronary sinus. Initially the reentry was anatomical, reaching the left atrium through the coronary sinus and returning through the septum and posterior interatrial bundles. However, after 5 cycles the wave found a shortcut at the most inferior of the interatrial bundles and a meandering spiral wave developed near the right inferior pulmonary vein. It 

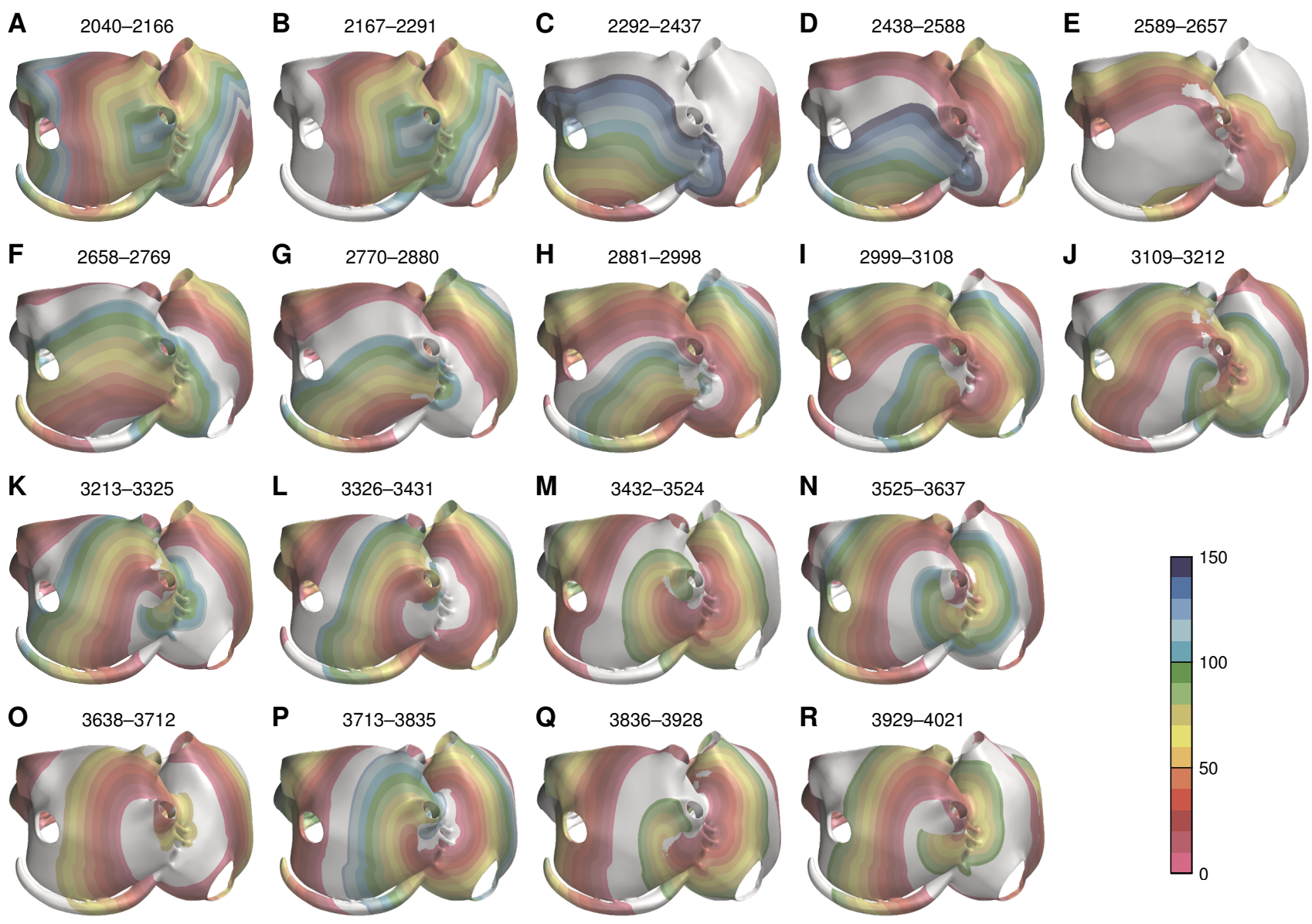

Figure 2. Example of AF initiation (pacing site 1, in the left atrial appendage, $101 \% g_{\mathrm{CaL}}$ ). The panels show 18 consecutive intervals during the simulation, as indicated on top of each panel in milliseconds from the start of the simulation. Colors show activation times; the color scale labels apply with respect to the start of each interval. The view is posterior. A: activation fronts of the last and pre-last stimuli. The pre-last stimulated wave is seen entering the coronary sinus from the left atrium. B: the last stimulated wave blocks at the connection between the left atrium and the coronary sinus and enters the latter through the right atrium. $\mathrm{C}-\mathrm{G}$ : the wave now crosses retrogradely from the coronary sinus to the left atrium and follows the same circuit 5 times. H-R: A spiral wave develops with its tip meandering near the right inferior pulmonary vein.

remained there during the rest of the simulation.

Variations in $g_{\mathrm{CaL}}$ significantly modified the effects of rapid pacing at this site. With $100 \% g_{\mathrm{CaL}}$, the last paced wave did not block at the connection between the left atrium and the coronary sinus and no AF was induced. With $102 \% g_{\mathrm{CaL}}$, AF initiation happened in the same way as in the $101 \%$ case, but after the spiral wave near the left inferior pulmonary vein developed it moved to the right atrium, where it remained near the inferior caval vein. A wave break at the crista terminalis induced a concurrent reentry near the superior caval vein.

Table 3 summarizes the results for all simulations in electrically remodeled atria. The table shows how long activity lasted beyond the last stimulus, which occurred at $2088 \mathrm{~ms}$ after the start of the simulation. In 8 of 30 simula- tions ( $27 \%$ ), activity lasted until the end of the simulation. In the others, activity lasted between 533 and 1417 ms after the last pacing pulse.

AF initiation succeeded most frequently with pacing in site 9 (right atrium near the sinus node) and site 1 (left atrial appendage).

In 30 simulations without electrical remodeling, activation never lasted more than $182 \mathrm{~ms}$ beyond the last pacing pulse.

\section{Discussion}

This study shows that in a structurally heterogeneous model AF can be induced by rapid pacing, demonstrating that intrinsic repolarization heterogeneity is not a require- 


\begin{tabular}{cccc}
\hline pacing & \multicolumn{3}{c}{$g_{\text {CaL }}$} \\
\cline { 2 - 4 } site & $100 \%$ & $101 \%$ & $102 \%$ \\
\hline 1 & 0.609 & $>8$ & $>8$ \\
2 & 0.702 & 0.552 & 0.536 \\
3 & 0.564 & 0.568 & $>8$ \\
4 & $>8$ & 1.417 & 1.398 \\
5 & 0.533 & 0.538 & 0.543 \\
6 & 0.578 & $>8$ & 0.572 \\
7 & 0.605 & 0.605 & 0.603 \\
8 & 0.543 & 0.539 & 0.550 \\
9 & $>8$ & $>8$ & $>8$ \\
10 & 0.591 & 0.603 & 0.591 \\
\hline
\end{tabular}

Table 3. Duration (in seconds) of activity after the last pacing pulse.

ment. In addition we found that small changes in $g_{\mathrm{CaL}}$ enabled or disabled AF initiation in 4 out of 30 simulations. Altogether, AF was initiated from 5 of the 10 pacing sites for some value of $g_{\mathrm{CaL}}$. However, electrical remodeling, involving a shortening of APD, was necessary: without this remodeling AF could not be induced with the same pacing protocol.

A shortcoming of this study is that the pacing protocol was designed to gently approach the refractory period of the electrically remodeled tissue, so that all stimuli were captured. In the non-remodeled simulations not all stimuli were captured. Thus, we cannot exclude that $\mathrm{AF}$ initiation would also be possible without electrical remodeling if a well-tuned pacing protocol is used.

Nevertheless, our results strongly suggest that homogeneous electrical remodeling can contribute to the duration of AF paroxysms and thus accelerate the development of structural remodeling and permanent AF.

The results also demonstrate that the success of an $\mathrm{AF}$ induction attempt is highly sensitive to $g_{\mathrm{CaL}}$, in a seemingly random fashion. This suggests that small variations in other physiological factors, such as autonomic tone, can also have a decisive effect on AF induction.

\section{Acknowledgements}

This work was granted access to HPC resources of TGCC-CEA and IDRIS under GENCI allocation 2018A0030307379. This work was supported by the French National Research Agency, grant reference ANR-10IAHU04-LIRYC.

\section{References}

[1] Haïssaguerre M, Jaïs P, Shah DC, Garrigue S, Takahashi A, Lavergne T, Hocini M, Peng JT, Roudaut R, Clémenty J. Spontaneous initiation of atrial fibrillation by ectopic beats originating in the pulmonary veins. N Engl J Med 1998; 339:659-666.

[2] Hocini M, Ho SY, Kawara T, Linnenbank AC, Potse M, Shah D, Jaïs P, Janse MJ, Haïssaguerre M, de Bakker JMT. Electrical conduction in canine pulmonary veins: Electrophysiological and anatomic correlation. Circulation 2002; 105:2442-2448.

[3] Schotten U, Verheule S, Kirchhof P, Goette A. Pathophysiological mechanisms of atrial fibrillation: A translational appraisal. Physiol Rev 2011;91:265-325.

[4] Krueger MW, Dorn A, Keller DUJ, Holmqvist F, Carlson J, Platonov PG, Rhode KS, Razavi R, Seemann G, Dössel O. In-silico modeling of atrial repolarization in normal and atrial fibrillation remodeled state. Med Biol Eng Comput 2013;51:1105-1119.

[5] Kneller J, Zou R, Vigmond EJ, Wang Z, Leon LJ, Nattel S. Cholinergic atrial fibrillation in a computer model of a twodimensional sheet of canine atrial cells with realistic ionic properties. Circ Res 2002;90:e73-e87.

[6] Potse M, Lankveld TAR, Zeemering S, Dagnelie PC, Stehouwer CDA, Henry RM, Linnenbank AC, Kuijpers NHL, Schotten U. P-wave complexity in normal subjects and computer models. J Electrocardiol 2016;49:545-553.

[7] Ho SY, Anderson RH, Sánchez-Quintana D. Atrial structure and fibres: morphologic bases of atrial conduction. Cardiovasc Res 2002;54:325-336.

[8] Ho SY, Cabrera JA, Sanchez-Quintana D. Left atrial anatomy revisited. Circ Arrhythm Electrophysiol 2012; 5:220-228.

[9] Chauvin M, Shah DC, Haïssaguerre M, Marcellin L, Brechenmacher $\mathrm{C}$. The anatomic basis of connections between the coronary sinus musculature and the left atrium in humans. Circulation 2000;101:647-652.

[10] Courtemanche M, Ramirez R, Nattel S. Ionic mechanisms underlying human atrial action potential properties: insights from a mathematical model. Am J Physiol Heart Circ Physiol 1998;275:H301-H321.

[11] Krause D, Potse M, Dickopf T, Krause R, Auricchio A, Prinzen FW. Hybrid parallelization of a large-scale heart model. In Keller R, Kramer D, Weiss JP (eds.), Facing the Multicore-Challenge II, volume 7174 of Lecture Notes in Computer Science. Berlin: Springer, 2012; 120-132.

[12] Gharaviri A, Verheule S, Eckstein J, Potse M, Kuklik P, Kuijpers NHL, Schotten U. How disruption of endoepicardial electrical connections enhances endo-epicardial conduction during atrial fibrillation. Europace 2017; 19:308-316.

Address for correspondence:
Mark Potse
CARMEN research team
Inria Bordeaux Sud-Ouest
200 Avenue de la vieille tour
33450 Talence, France
mark@potse.nl 